\title{
The Critical Role of Long Noncoding RNA in Osteogenic Differentiation of Human Bone Marrow Mesenchymal Stem Cells
}

\author{
Xiaoling Qiu, ${ }^{1}$ Bo Jia, ${ }^{2}$ Xiang Sun, ${ }^{2}$ Weitao $\mathrm{Hu}^{2}{ }^{2}$ Hongxing $\mathrm{Chu},{ }^{2}$ \\ Shuaimei $\mathrm{Xu},{ }^{1}$ and JianJiang $\mathrm{Zhao}^{2}$ \\ ${ }^{1}$ Department of Endodontics, Stomatological Hospital of Southern Medical University and \\ Guangdong Provincial Stomatological Hospital, Guangzhou 510280, China \\ ${ }^{2}$ Department of Oral Surgery, Stomatological Hospital of Southern Medical University and \\ Guangdong Provincial Stomatological Hospital, Guangzhou 510280, China
}

Correspondence should be addressed to JianJiang Zhao; jianjiang_zhao@163.com

Received 14 November 2016; Accepted 2 April 2017; Published 27 April 2017

Academic Editor: Milena Fini

Copyright (C) 2017 Xiaoling Qiu et al. This is an open access article distributed under the Creative Commons Attribution License, which permits unrestricted use, distribution, and reproduction in any medium, provided the original work is properly cited.

\begin{abstract}
Objective. Long noncoding RNAs (lncRNAs) have been demonstrated to regulate many biological processes including differentiation. However, their role in osteogenic differentiation was poorly known. Materials and Methods. In this study, we first globally profiled the differentially expressed lncRNAs and mRNAs during osteogenic differentiation of human bone marrow mesenchymal stem cells (hBMMSCs). Bioinformatics analysis was performed to further analyze these significantly changed molecules. Then the role of lncRNA ENST00000502125.2 in the osteogenic differentiation was determined. Results. A number of lncRNAs and mRNAs were significantly differentially expressed during hBMMSC osteogenic differentiation. Among them, 433 lncRNAs and 956 mRNAs were continuously upregulated, while $232 \mathrm{lncRNAs}$ and 229 mRNAs were continuously downregulated. Gene Ontology and KEGG (Kyoto Encyclopedia of Genes and Genomes) analysis showed that carbohydrate derivative binding and complement and coagulation cascades were most correlated molecular function and pathway, respectively. Downregulation of lncRNA ENST00000502125.2 promoted the osteogenic differentiation of hBMMSCs, and opposite results were found when lncRNA ENST00000502125.2 was upregulated. Conclusions. lncRNAs play a critical role in the osteogenic differentiation of hBMMSCs and targeting lncRNA ENST00000502125.2 might be a promising strategy to promote osteogenic differentiation.
\end{abstract}

\section{Introduction}

In the craniomaxillofacial skeleton, bone defect is still a major and challenging health concern. Its size can vary from the very small periodontal defects to the large segmental defects [1]. Efficacious bone tissue engineering requires a combination of three key factors including stem cells, growth factors, and scaffolds [2]. Human mesenchymal stem cells (hMSCs) are adult stem cells that can be isolated from adult tissues such as bone marrow, adipose tissues, and dental pulp [3, 4]. In addition to colony formation and self-renewal capability, hMSCs have the capacity to differentiate into a number of cell types such as osteoblasts, adipocytes, chondrocytes, and neural-like cells $[5,6]$. Moreover, the immunosuppressive potential of hMSCs is important for the treatment of graftversus-host diseases [7] These intrinsic properties of hMSCs make them an ideal cell source for both cellular therapy and regenerative medicine.

Long noncoding RNAs (lncRNAs) are a class of nonprotein-coding transcripts with a length of more than 200 nucleotides [8]. Similarly to microRNAs, lncRNAs have been demonstrated to play a central role in regulating many important biological processes such as cell proliferation, differentiation, migration, and development $[9,10]$. Mutations and deregulations of lncRNAs link to the initiation and progression of diverse human diseases ranging from cardiovascular 
diseases to cancer [11]. Various studies reported that lncRNAs are important for the osteogenic differentiation of MSCs. Downregulation of lncRNA MEG3 significantly suppressed the osteogenic potential of MSCs, and opposite findings were detected when IncRNA MEG3 was overexpressed. In addition, its regulatory effects on osteogenic differentiation were achieved by partly activating BMP4 [12]. Similarly, lncRNA MIR31HG inhibition promoted the osteogenic differentiation of human adipose-derived stem cells (hASCs) in either normal or inflammatory environment by regulating $N F-\kappa B$ signalling pathway, indicating lncRNA MIR31HG- NF- $\kappa \mathrm{B}$ axis is critical for the osteogenic differentiation of hASCs [13].

However, the function of lncRNAs in the osteogenic differentiation of human bone marrow MSCs (hBMMSCs) remains poorly known. Therefore, the purpose of the current study was global profiling of the changes of mRNA and lncRNA during the osteogenic differentiation of hBMMSCs and to determine the role of lncRNA ENST00000502125.2 in the process of osteogenesis.

\section{Materials and Methods}

2.1. Cell Culture. HBMMSCs were purchased from ScienCell Research Laboratories (Cat. number 7500; Carlsbad, CA, USA). The hBMMSCs were maintained with Mesenchymal Stem Cell Medium (ScienCell, Carlsbad, CA, USA) at $37^{\circ} \mathrm{C}$ in a humidified atmosphere of $95 \%$ air and 5\% CO2. For the osteogenic differentiation of hBMMSCs, the cells were cultured in osteogenic medium containing $10 \mathrm{mmol} / \mathrm{L} \beta$-glycerol phosphate, $50 \mu \mathrm{g} / \mathrm{mL}$ ascorbic acid, and 10-7 mol/L dexamethasone.

2.2. Real-Time PCR. Total RNA was isolated from hBMMSCs using TRIzol (Invitrogen, Carlsbad, CA, USA) according to the manufacturer's instructions. First-strand cDNA was synthesized from $2 \mu \mathrm{g}$ RNA using the SuperScript ${ }^{\circledR}$ III Reverse Transcriptase (Invitrogen). Then the cDNA levels were amplified and quantified with SYBR Green PCR master mix (Applied Biosystems, Foster City, CA, USA). The PCR reaction was performed using the 7900HT Fast Real-Time PCR System (Applied Biosystems). Gene expression was normalized against glyceraldehyde-3-phosphate dehydrogenase (GAPDH). The primer sequence was summarized in Supplementary Table 1 (in Supplementary Material available online at https://doi.org/10.1155/2017/5045827).

\subsection{Chip Arrays. Human $4 \times 180 \mathrm{~K} \operatorname{lncRNA}$ arrays contained} $37581 \mathrm{lncRNAs}$ and 34235 mRNAs. Total RNA was obtained as described above. Approximately $200 \mathrm{ng}$ of total RNA from each sample was used for the microarray analyses. Briefly, followed by spiking with an RNA Spike-In Kit with one color (Agilent), total RNA was reversely transcribed into cDNA and then converted into Cyanine-3 labeled cRNA. Cyanine-3labeled cRNA sample was fragmented and then hybridized at $65^{\circ} \mathrm{C}$ for $17 \mathrm{~h}$ using an Agilent Gene Expression Hybridization Kit in hybridization chamber gasket slides (Agilent). After hybridization, the microarrays were washed with an Agilent Wash Buffer kit (Agilent) and scanned with an Agilent microarray scanner. The resulting images were analyzed using Agilent's Feature Extraction software v10.7 and Agilent GeneSpring.

2.4. Gene Function Analysis. To investigate the potential functions of these lncRNAs, the predicted target genes were input into the Database for Annotation, Visualization and Integrated Discovery (DAVID; http://david.abcc.ncifcrf.gov/), which utilized Gene Ontology (GO) to identify the molecular function. In addition, KEGG (Kyoto Encyclopedia of Genes and Genomes) database (http://www.genome.ad.jp/kegg/) was employed to analyze the potential functions of these target genes in the pathways. Lower $p$ value indicates that the correlation is more significant. The cut-off $p$ value is 0.05 .

2.5. Lentivirus Construction and Transfection. The lncRNA ENST00000502125.2-targeting (5'-GGTTAATCCACAGTTAGGAGCTTCCTGTCAGACTCCTAACTGTGGATTAACCTTTTT- $\left.3^{\prime}\right)$ /overexpression (5' ${ }^{\prime}$ TTCCCCATCCACAGAAATGGT-3') oligonucleotide sequences were cloned into the lentiviral vectors. The mock control consisted of a scrambled sequence with no homology to any gene in the human genome. Recombinant lentiviral vectors and packaging vectors were then transfected into $293 \mathrm{~T}$ cells. The supernatant containing lentiviruses was harvested $72 \mathrm{~h}$ after transfection. The lentiviruses were then purified by ultracentrifugation, and the titer of lentiviruses was determined. Human BMMSCs were infected with the lentiviruses at a multiplicity of infection of 50 .

2.6. ALP Staining. About $2 \times 10^{5}$ cells/plate were seeded in six-well plates and subjected to osteogenic differentiation induction when the cell density reached $70-80 \%$ confluence. ALP staining was performed with an ALP staining kit (SigmaAldrich) at day 7 according to the manufacturer's protocols. Briefly, the induced cells were fixed in $4 \%$ formaldehyde and incubated with a substrate buffer at room temperature (RT) for 30 minutes. The reaction was terminated by removing the substrate solution and washing with PBS. The staining results were observed under microscope.

2.7. Alizarin Red S Staining. About $2 \times 10^{5}$ cells/plate were seeded in six-well plates and subjected to osteogenic differentiation induction when the cell density reached $70-80 \%$ confluence. The induced cells at day 21 were gently washed with PBS twice and then fixed in 4\% formaldehyde for $30 \mathrm{~min}$ at RT. Then cells were washed with distilled water three times, stained with $2 \%$ Alizarin Red S for $30 \mathrm{~min}$ at RT, and washed with distilled water to remove the remaining staining. The staining results were observed under microscope.

2.8. Statistical Analysis. The data were analyzed and expressed as means \pm standard deviation. All the experiments were performed in triplicate and repeated three times. The threshold value we used to screen differentially expressed lncRNAs and mRNAs was a fold change 22.0 . Differences between two groups or more than two groups were evaluated 

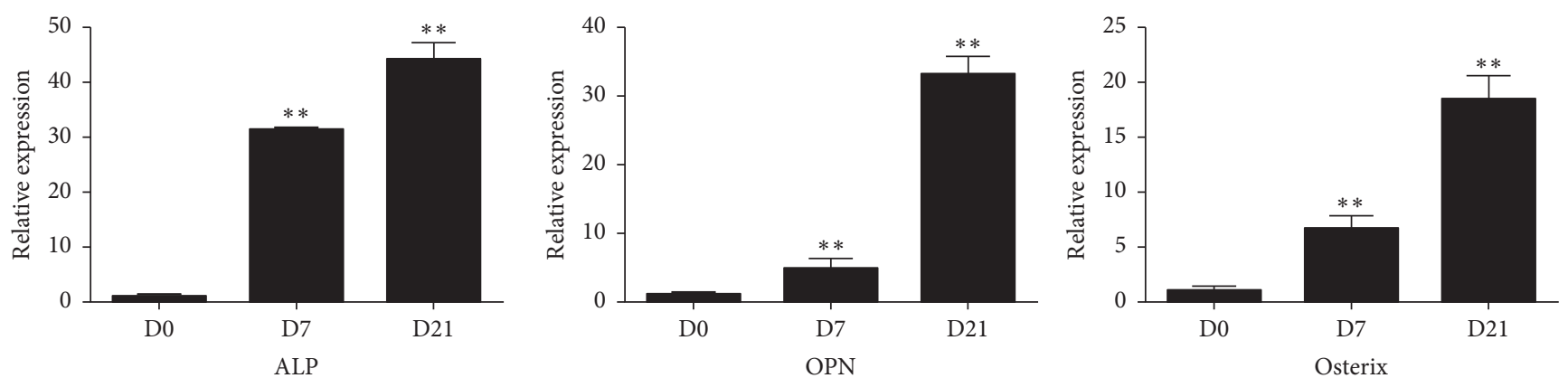

(a)

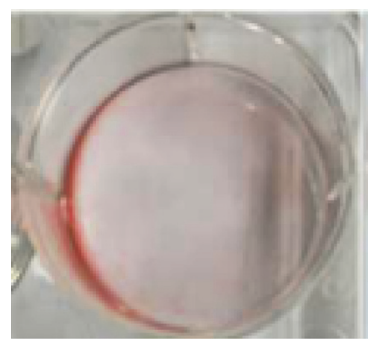

D0

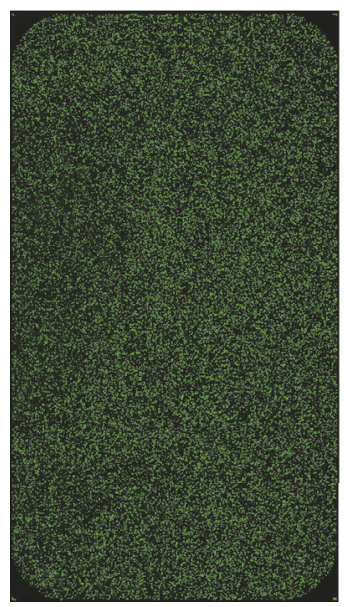

D0

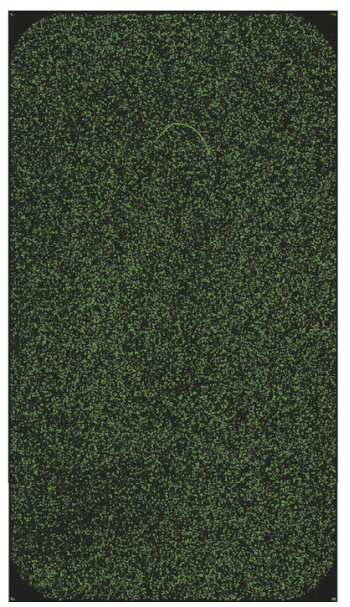

D7

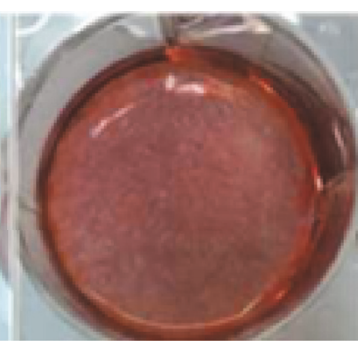

D14

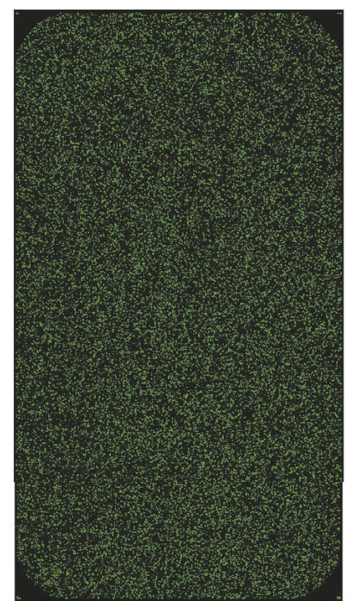

D14

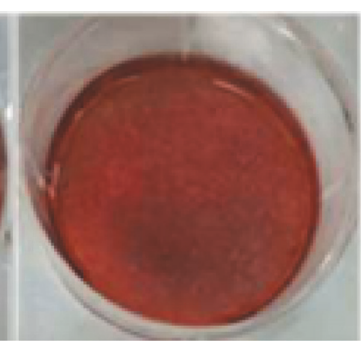

D21

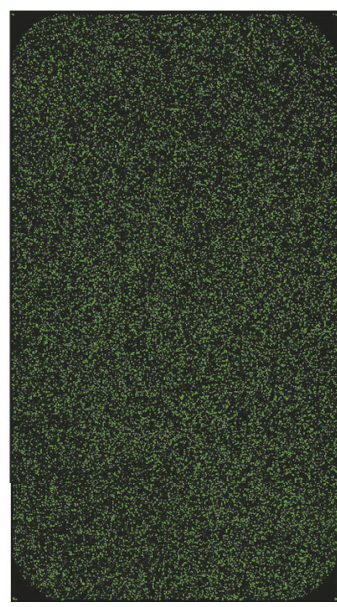

D21

(c)

FIGURE 1: (a) The expression levels of osteogenic markers ALP, OPN, and Osterix were significantly increased following osteogenic induction $\left({ }^{* *} p<0.01\right)$. (b) The staining intensity of Alizarin Red S was increased following osteogenic induction. (c) Microarray analysis of gene expression during osteogenic differentiation of hBMMSCs.

for statistical significance by the independent $t$-test and OneWay ANOVA, respectively, using SPSS v21.0 software (SPSS Inc., Chicago, IL). Statistical significance was set at $p<0.05$.

\section{Results}

3.1. The Differentially Expressed $\ln c R N A / m R N A$ during the Osteogenic Differentiation of hBMMSCs. The expression levels of osteogenic markers including ALP, OPN, and Osterix as well as Alizarin Red S staining intensity were significantly increased after in vitro induction of osteogenic differentiation (Figures 1(a) and 1(b)).
The results of microarray assay were shown in Figure 1(c). The analysis revealed that $923 \operatorname{lncRNA}, 1393 \operatorname{lncRNA}$, and 1338 lncRNA were significantly increased ( $>2$ fold) in cells cultured in osteogenic medium at D7, D14, and D21, respectively, compared to those at D0, while 993 lncRNA (D7), 3843 lncRNA (D14), and 3688 lncRNA (D21) were remarkably decreased ( $\leq 2$ fold). Similarly, compared with the controls (D0), 1462 mRNA (D7), 4093 mRNA (D14), and 3354 mRNA (D21) were significantly upregulated while 953 mRNA (D7), 2236 mRNA (D14), and 1923 mRNA (D21) were downregulated more than two times. The hierarchical cluster analysis revealed the differentially expressed lncRNAs (Figure 2(a)) and mRNAs (Figure 2(b)) during the osteogenic 
TABLE 1: The consistent upregulated lncRNAs in D7 group, D14 group, and D21 group compared to D0 group.

\begin{tabular}{|c|c|c|c|c|c|c|}
\hline \multirow{2}{*}{ Probe name } & \multicolumn{3}{|c|}{ Fold change } & \multirow{2}{*}{ lncRNA ID } & \multirow{2}{*}{ Chromosome } & \multirow{2}{*}{ Strand } \\
\hline & D7 versus D0 & D14 versus D0 & D21 versus D0 & & & \\
\hline p4114 & 21.62478164 & 11.60060386 & 25.92556994 & ENST00000418923.1 & 13 & - \\
\hline p38401_v4 & 30.86834436 & 39.89716139 & 47.83972529 & ENST00000390168.4 & 11 & - \\
\hline p35386_v4 & 8.850828267 & 13.50510134 & 8.569022202 & ENST00000524338.1 & 8 & - \\
\hline p33814 & 10.7500145 & 19.33207243 & 15.16926732 & uc021wsx.1 & 3 & - \\
\hline p34143_v4 & 17.84687532 & 21.39256843 & 22.59689433 & ENST00000428066.1 & 11 & - \\
\hline p20779 & 11.0580876 & 8.572849402 & 12.7458369 & TCONS_00004149 & 2 & - \\
\hline p29982 & 14.36987842 & 18.31301009 & 19.1400538 & TCONS_00015010 & 8 & - \\
\hline p34144_v4 & 9.448857515 & 11.1571628 & 12.01925275 & ENST00000447298.1 & 11 & - \\
\hline p13783 & 29.95472326 & 22.41719711 & 21.2711142 & ENST00000507217.1 & 5 & + \\
\hline p41550_v4 & 24.25949023 & 36.60054941 & 28.7447275 & XR_242466.1 & 8 & - \\
\hline p15703 & 16.07037289 & 23.13735751 & 15.39810811 & ENST00000521483.1 & 8 & - \\
\hline p35387_v4 & 13.86215164 & 20.48568771 & 12.3106551 & ENST00000518943.1 & 8 & - \\
\hline p33593 & 8.765900177 & 18.72201809 & 18.08884202 & ENST00000454645.1 & 9 & - \\
\hline p1239 & 8.87593437 & 29.58522619 & 15.2389718 & ENST00000423943.1 & 1 & + \\
\hline p26385 & 8.374898467 & 9.089043638 & 11.37470244 & uc003tcq.1 & 7 & - \\
\hline p23967 & 15.48067958 & 19.56789008 & 13.49101323 & TCONS_00015014 & 8 & - \\
\hline p23964 & 10.21976098 & 12.64248095 & 12.07873525 & TCONS_00015011 & 8 & - \\
\hline p15702 & 15.66396998 & 21.61260698 & 15.13024559 & ENST00000523786.1 & 8 & - \\
\hline p23883 & 11.98005378 & 16.83343272 & 9.64895952 & TCONS_00013634 & 7 & + \\
\hline p15704 & 11.4736364 & 19.16351897 & 11.40714697 & ENST00000523664.1 & 8 & - \\
\hline
\end{tabular}

differentiation of hBMSCs. The red and the green shades indicated the expression above and below the relative expression. Among these significantly differentially expressed lncRNA/ mRNA, 433 lncRNAs and 956 mRNAs were continuously upregulated. While $232 \mathrm{lncRNAs}$ and $229 \mathrm{mRNAs}$ were continuously downregulated. The representative consistent upregulated/downregulated lncRNAs and mRNAs were summarized in Tables 1-4.

Then we chose four consistently upregulated/downregulated $\operatorname{lncRNAs}$ for $\mathrm{qRT}$-PCR validation. qRT-PCR results revealed the expression levels of ENST00000523786.1 and ENST00000436715.1 were significantly upregulated during hBMMSC osteogenic differentiation $(p<0.01)$, while ENST00000532315.1 and HIT000218960 expression were downregulated $(p<0.01)$ (Figure 2(c)). The qRT-PCR results further corroborated that our microarray data was convincing.

3.2. Bioinformatics Analysis of Differentially Expressed lncRNAs and $m R N A$ s during Osteogenesis of hBMMSCs. Six hundred and eighty significantly differentially expressed mRNAs were enriched in biological processes during osteogenic differentiation of hBMMSCs and system development hit the highest score. As regards cellular component, there were 702 differentially expressed mRNAs. Among them, the extracellular matrix (ECM) contained most abundant differentially expressed genes. Six hundred and thirty-one differentially expressed mRNAs were enriched in molecular function and carbohydrate derivative binding had the highest score (Figures 3(a)-3(c)).
KEGG analysis showed that there were 427 differentially expressed mRNAs enriched in 31 biological pathways during osteogenesis of hBMMSCs. The pathway with highest score was complement and coagulation cascades, which included 21 differentially expressed genes (Figure 4).

\subsection{The Effects of IncRNA ENST00000502125.2 Upregulation/} Downregulation on the Osteogenic Differentiation of hBMMSCs. The expression level of lncRNA ENST00000502125.2 was significantly decreased during osteogenic differentiation of hBMMSCs (Figure 5(a)).

The target sequences we designed could upregulate or downregulate IncRNA ENST00000502125.2 with high efficiency (Figures 5(b) and 5(c)). Our results showed that the ALP staining intensity was significantly stronger in hBMMSCs subjected to lncRNA ENST00000502125.2 downregulation. In addition, the number of nodules was significantly more in the lncRNA ENST00000502125.2 knockdown cells. However, the staining intensities of ALP and Alizarin Red S assays were weaker when lncRNA ENST00000502125.2 was overexpressed (Figures 5(d) and 5(e)).

\section{Discussion}

LncRNAs had long been considered as nonfunctional junk [14]. However, recent studies showed that lncRNAs play a central role in regulating a number of biological processes including, but not limited to, transcription regulation, epigenetic modification, DNA methylation, and histone modification [15]. Deregulation of lncRNAs has been associated with 


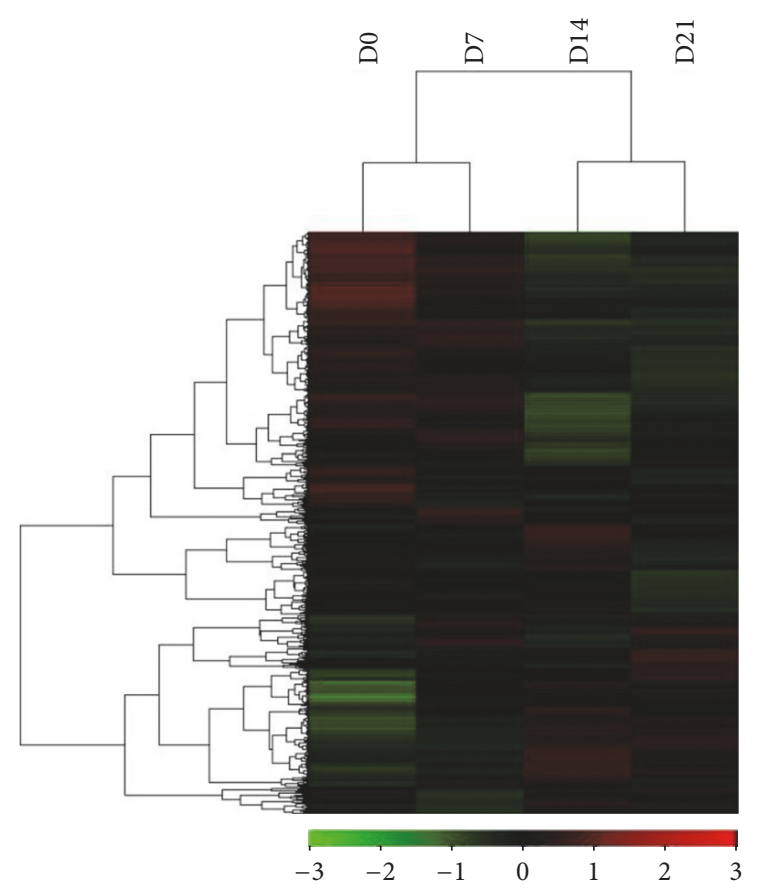

(a)
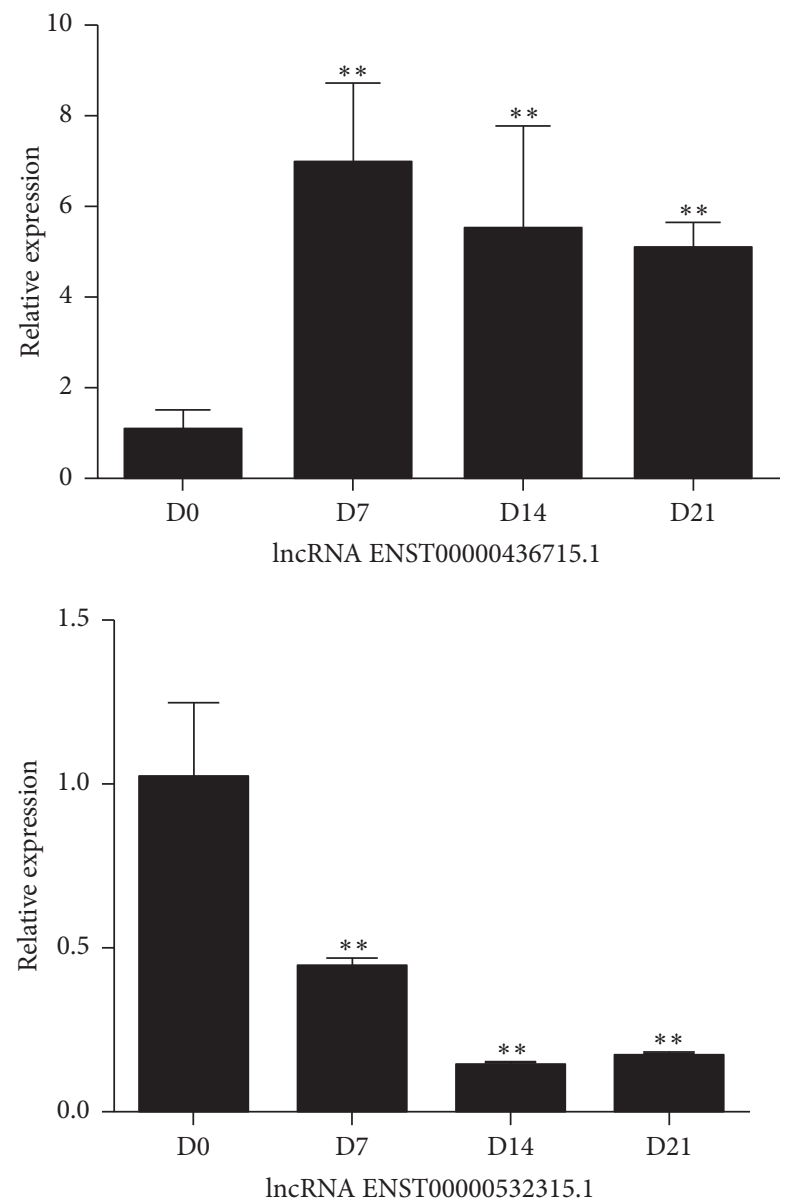

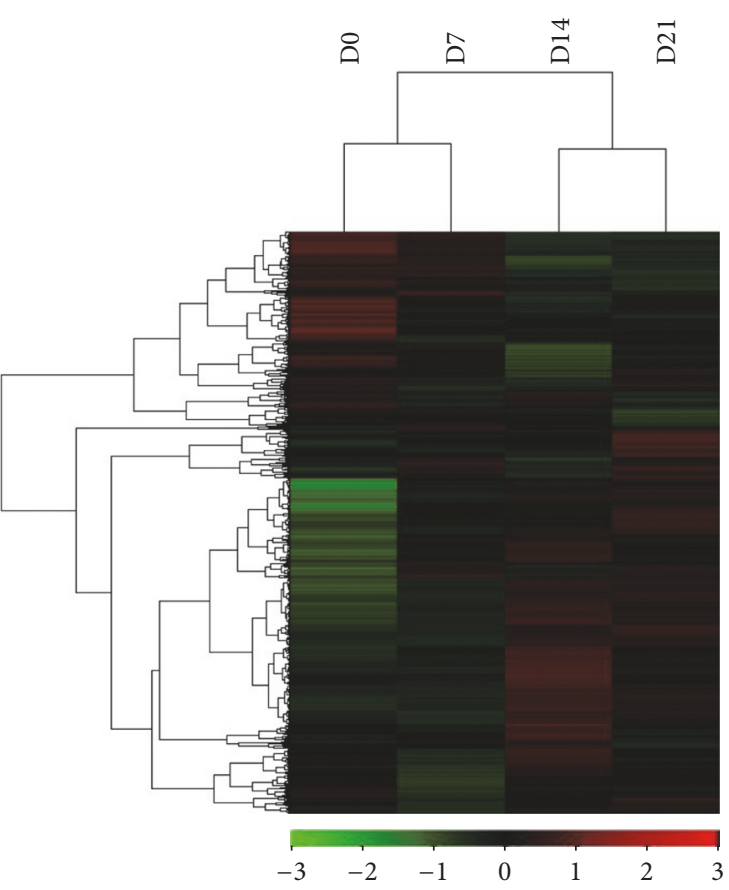

(b)
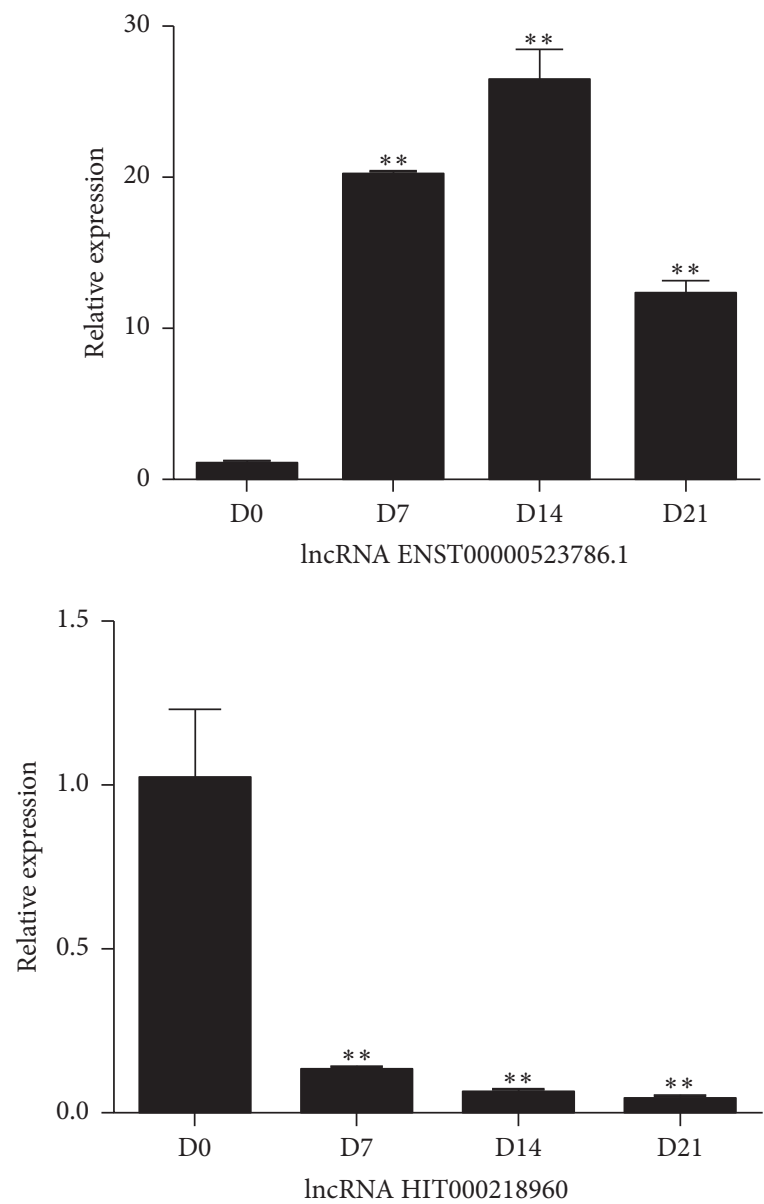

(c)

Figure 2: (a) The hierarchical cluster analysis revealed the differentially expressed lncRNAs during the osteogenic differentiation of hBMMSCs. (b) The hierarchical cluster analysis revealed the differentially expressed mRNAs during the osteogenic differentiation of hBMMSCs. (c) qPCR validation of the significantly different expressed lncRNAs during the osteogenic differentiation of hBMMSCs. ${ }^{* *}$ indicates $p<0.01$. 


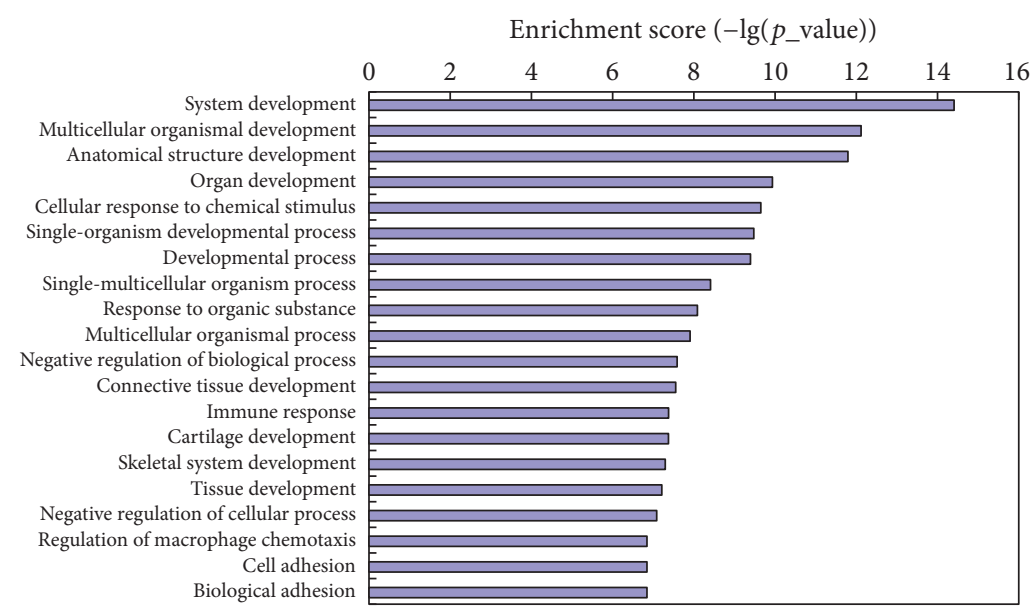

(a)

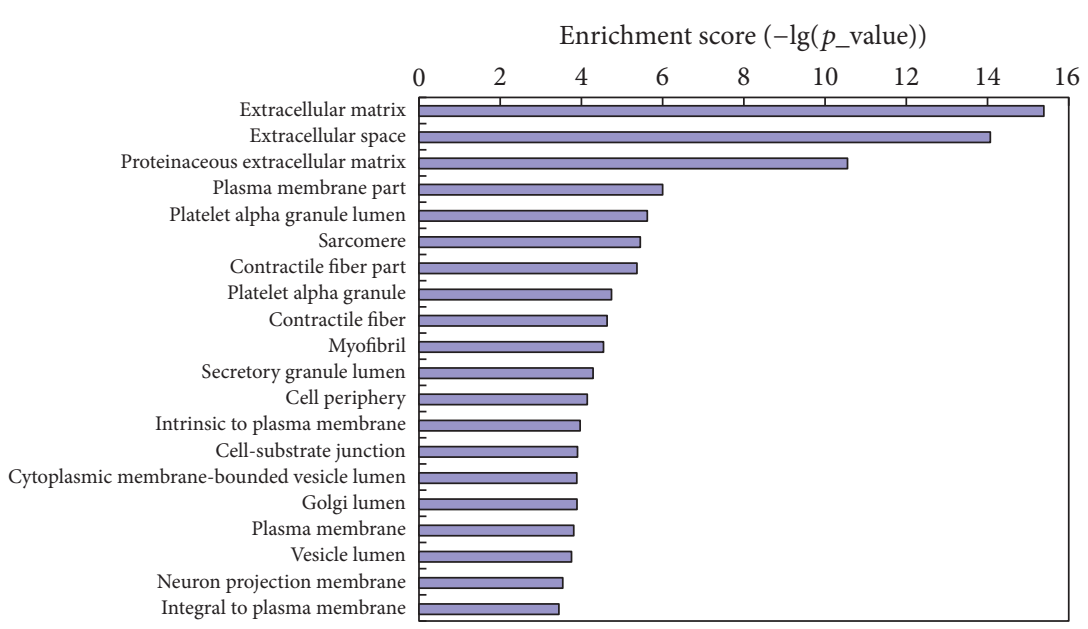

(b)

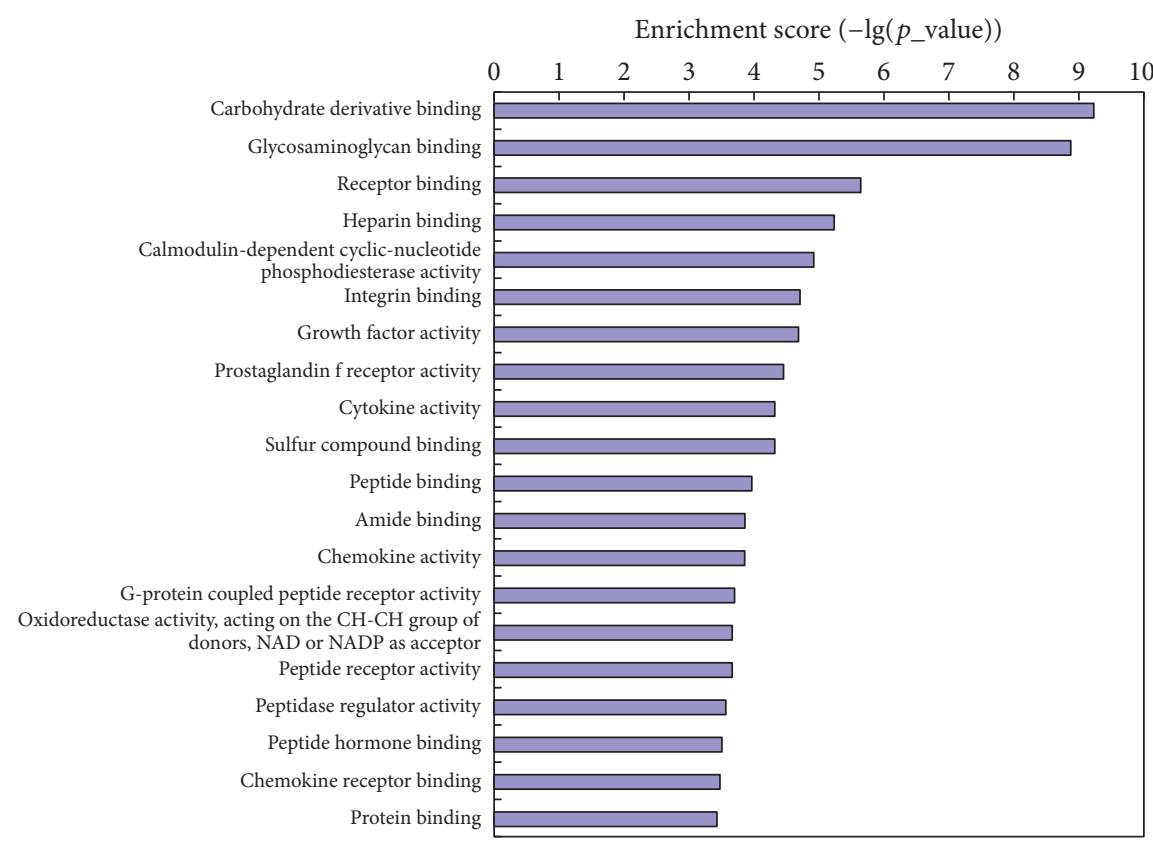

(c)

FIGURE 3: GO analysis of the significantly affected biological process, cellular component, and molecular function during osteogenic differentiation of hBMMSCs. 
TABLE 2: The larger continuous downregulated lncRNA in D7 group, D14 group, and D21 group compared to D0 group.

\begin{tabular}{|c|c|c|c|c|c|c|}
\hline \multirow{2}{*}{ Probe name } & \multicolumn{3}{|c|}{ Fold change } & \multirow{2}{*}{$\operatorname{lncRNA}$ ID } & \multirow{2}{*}{ Chromosome } & \multirow{2}{*}{ Strand } \\
\hline & D7 versus D0 & D14 versus D0 & D21 versus D0 & & & \\
\hline p26805 & -6.180586296 & -18.31712024 & -21.76631127 & eHIT000015952 & 3 & - \\
\hline p26850 & -3.162097824 & -7.896981442 & -5.113394731 & HIT000062015 & 5 & + \\
\hline p6751 & -3.075056887 & -9.43623037 & -4.115732163 & ENST00000567574.1 & 17 & - \\
\hline p11958 & -3.095430977 & -11.50752842 & -4.973522833 & ENST00000464242.1 & 3 & + \\
\hline p43709_v4 & -3.119120027 & -6.225875253 & -6.275615097 & NR_109889.1 & 20 & - \\
\hline p18116 & -3.243406388 & -5.670092378 & -5.470304342 & TCONS_00018160 & 10 & + \\
\hline p33827 & -3.553411085 & -4.48591536 & -4.526856894 & uc021zin.1 & 6 & + \\
\hline p41682_v4 & -3.08170091 & -3.169656935 & -6.44910429 & XR_242518.2 & 9 & + \\
\hline p24751 & -3.332997418 & -8.352157977 & -4.184503053 & XR_109875.3 & 1 & - \\
\hline p25066 & -3.147224208 & -7.23319858 & -5.77391016 & NR_026880.1 & 17 & + \\
\hline p7544 & -3.526526302 & -5.765265908 & -7.295186192 & ENST00000574526.1 & 17 & + \\
\hline p601 & -3.396662015 & -7.265009931 & -6.836209784 & ENST00000413035.1 & 1 & - \\
\hline p5379 & -3.951455716 & -6.197452204 & -4.222822011 & ENST00000561344.1 & 15 & - \\
\hline p5378 & -3.263970006 & -13.03398613 & -8.189183014 & ENST00000502125.2 & 15 & - \\
\hline p2545 & -4.012409632 & -8.23404424 & -5.849793103 & ENST00000532315.1 & 11 & - \\
\hline p26487 & -3.084674582 & -4.190874299 & -4.371006429 & uc004aaw.1 & 9 & - \\
\hline p34220_v4 & -4.329673813 & -3.902414327 & -4.992448343 & ENST00000564531.1 & 12 & - \\
\hline p7506 & -3.071148345 & -14.10807749 & -5.324648534 & ENST00000584660.1 & 17 & + \\
\hline p37929_v4 & -3.347180055 & -5.438810651 & -5.171230814 & ENST00000603042.1 & 6 & - \\
\hline
\end{tabular}

TABLE 3: The consistent upregulated mRNA in D7 group, D14 group, and D21 group compared to D0 group.

\begin{tabular}{lcccc}
\hline Probe name & & Fold change & & \\
& D7 versus D0 & D14 versus D0 & D21 versus D0 & TMEMA ID \\
\hline A_23_P157007 & 20.93078768 & 46.88202561 & 48.44960505 & SULT1E1 \\
A_23_P155786 & 40.92369481 & 33.41643908 & 40.18324588 & CADM3 \\
A_33_P3421923 & 45.38015715 & 161.8576338 & 737.750768 & IBSP \\
A_23_P144549 & 49.02268505 & 21.60574241 & 31.28494343 & CSTA \\
A_23_P4114 & 21.69170424 & 65.94353401 & 34.50429618 & CFD \\
A_23_P119562 & 23.82145451 & 36.79416271 & 50.64949923 & NEBL \\
A_24_P398147 & 36.09050187 & 42.0693231 & 206.0673461 & OMD \\
A_23_P94397 & 69.98586179 & 126.4123144 & 69.37444886 & 59.15291665 \\
A_24_P208436 & 30.66909159 & 52.38832752 & 30.92324555 & PDE1A \\
A_23_P363778 & 25.62987078 & 54.96002289 & 278.1804069 & FRZB \\
A_23_P52761 & 80.20879313 & 39.88630678 & 114.5446685 & MMP7 \\
A_23_P82990 & 75.35249622 & 66.33124962 & 296.7605893 & OGN \\
A_24_P48723 & 62.3488783 & 46.0152525 & 125.7786547 & PTGIS \\
A_33_P3361636 & 65.19427159 & 149.0955822 & 186.8343613 & MGP \\
A_23_P81158 & 43.72592716 & 109.8552875 & 24.81066209 & ADH1C \\
A_33_P3252286 & 27.80470836 & 197.9730171 & 223.5632762 & CRLF1 \\
A_23_P307310 & 24.53975993 & 22.28423515 & 35.02566896 & ACAN \\
A_24_P264943 & 154.5977058 & 192.3886928 & COMP \\
A_24_P330263 & 43.2923737 & 32.38378473 & EDNRB \\
\hline
\end{tabular}

the progression of many diseases such as cancer and cardiovascular diseases $[16,17]$.

Previous study have screened the expression profile of lncRNAs during osteogenic differentiation of MSCs [18]. However, the role of lncRNAs in osteogenesis remains poorly known. In this study, a number of novel lncRNAs and
mRNAs especially those consistently deregulated might regulate hBMMSC osteogenic differentiation. For instance, both leukemia inhibitory factor (LIF) and frizzled-related protein (FRZB) are critical for osteogenesis [19, 20]. Real-time PCR was performed to validate our microarray findings. The reason we chose ENST00000523786.1, ENST00000436715.1, 
TABLE 4: The consistent downregulated mRNA in D7 group, D14 group, and D21 group compared to D0 group.

\begin{tabular}{|c|c|c|c|c|}
\hline \multirow{2}{*}{ Probe name } & \multicolumn{3}{|c|}{ Fold change } & \multirow{2}{*}{ mRNA ID } \\
\hline & D7 versus D0 & D14 versus D0 & D21 versus D0 & \\
\hline A_33_P3421178 & -4.978203478 & -6.689279838 & -6.73293016 & ENST00000552367 \\
\hline A_33_P3347241 & -3.234257846 & -8.689836302 & -10.18643693 & ENST00000420598 \\
\hline A_23_P430658 & -4.081348909 & -5.123030497 & -4.953099079 & HEYL \\
\hline A_24_P122137 & -9.239361785 & -4.534924085 & -5.997598307 & LIF \\
\hline A_23_P358709 & -3.492627299 & -6.212881338 & -7.058185271 & AHRR \\
\hline A_33_P3240951 & -6.366913923 & -7.306438093 & -6.10226131 & DPF3 \\
\hline A_33_P3357658 & -6.362807169 & -9.205441781 & -12.61825188 & HMGA2 \\
\hline A_23_P19754 & -4.978203478 & -6.689279838 & -6.73293016 & CPA4 \\
\hline A_33_P3338693 & -10.83785842 & -9.210934927 & -11.71527326 & SNAP25 \\
\hline A_21_P0010759 & -3.730747446 & -9.78227973 & -5.089176405 & ENST00000458145 \\
\hline A_33_P3281667 & -3.873687841 & -5.554700388 & -5.14477388 & CNTNAP3B \\
\hline A_24_P160401 & -7.369096775 & -4.833372717 & -4.539895532 & CDCP1 \\
\hline A_33_P3422728 & -3.864556879 & -6.005084014 & -4.89254934 & CNTNAP3 \\
\hline A_23_P106194 & -5.131831246 & -4.991628029 & -4.329195734 & FOS \\
\hline A_33_P3325897 & -3.057861576 & -13.01790152 & -4.632399252 & MPL \\
\hline A_24_P50368 & -3.790519797 & -12.29526394 & -9.281170737 & BLID \\
\hline A_33_P3321372 & -3.234257846 & -8.689836302 & -10.18643693 & ENST00000377653 \\
\hline A_33_P3356392 & -3.256778088 & -5.440437604 & -8.336994595 & RGL3 \\
\hline A_33_P3265467 & -5.491535497 & -6.710400621 & -4.29031983 & ENST00000374375 \\
\hline A_33_P3263518 & -4.855417658 & -6.782055075 & -5.473469073 & ENST00000298953 \\
\hline
\end{tabular}

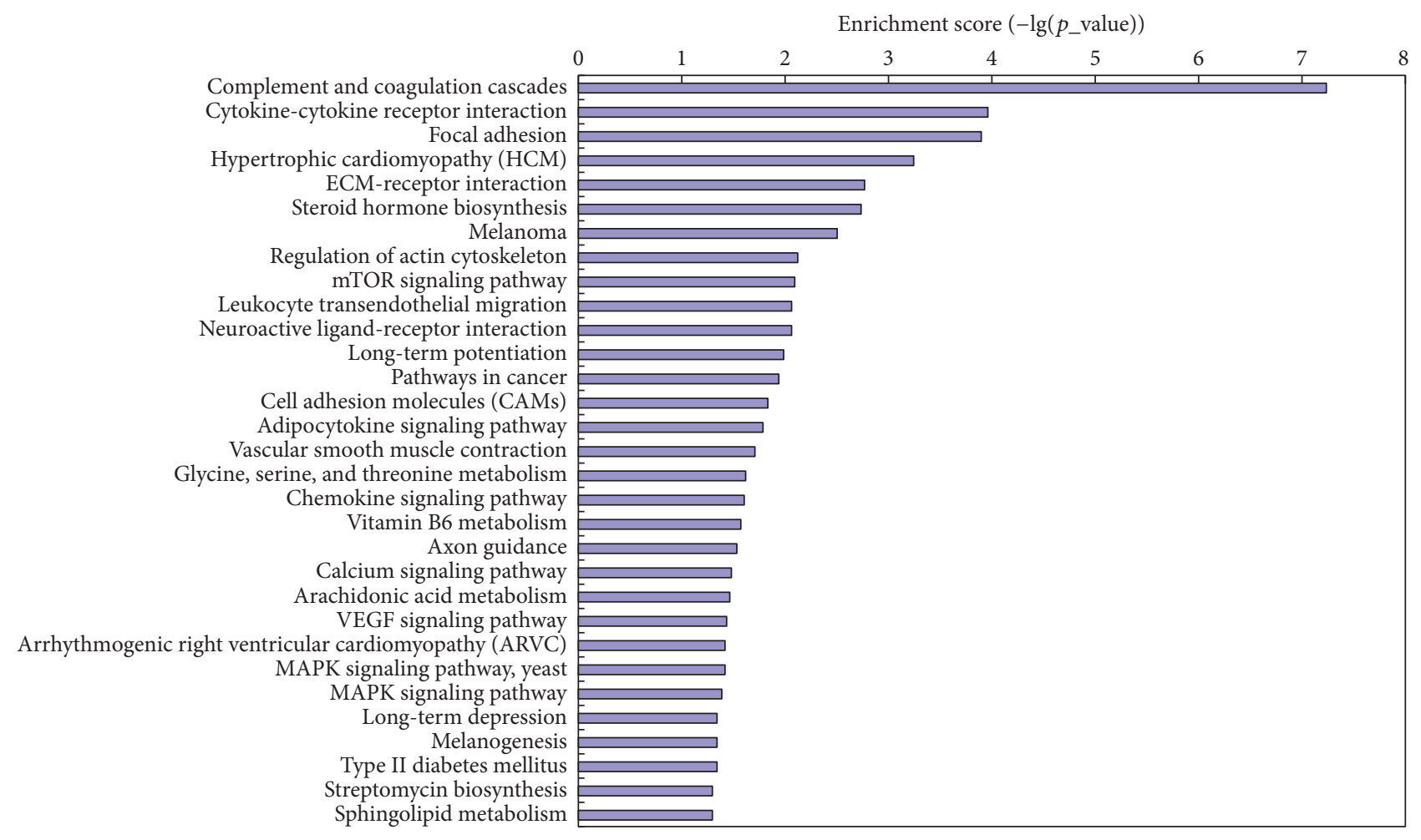

FIGURE 4: KEGG analysis of the significantly affected biological pathways during osteogenic differentiation of hBMMSCs. 


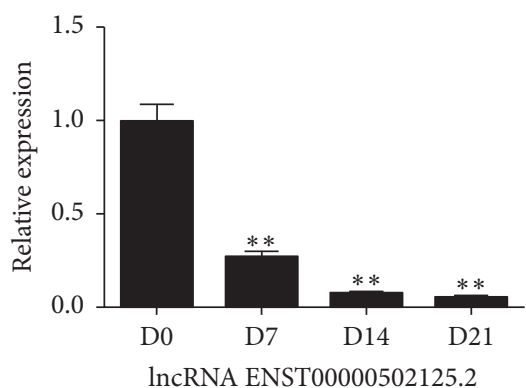

(a)

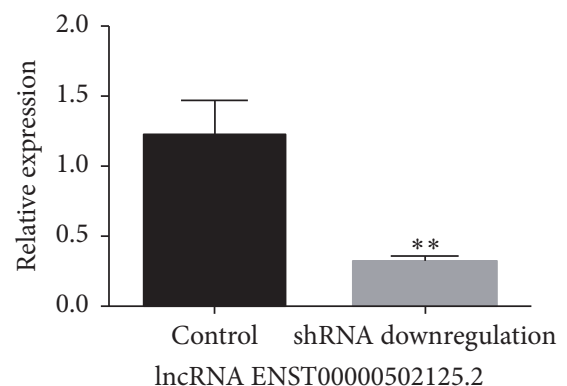

(b)

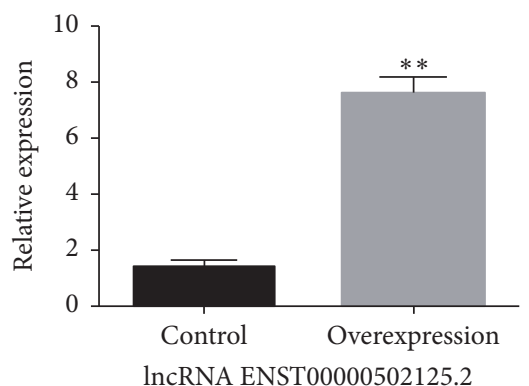

(c)

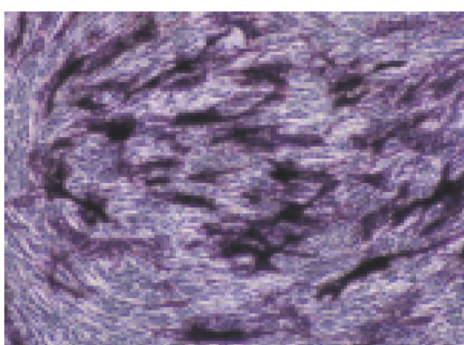

Control

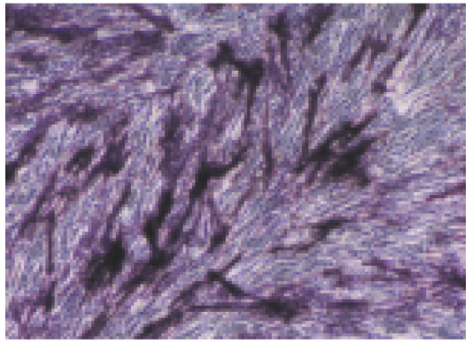

Control

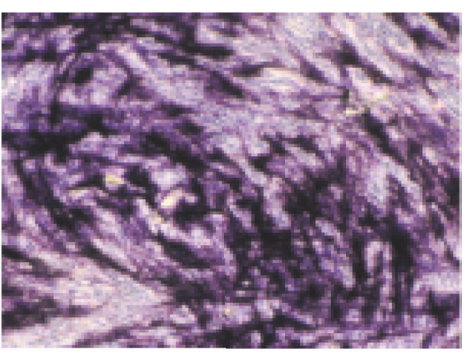

shRNA downregulation

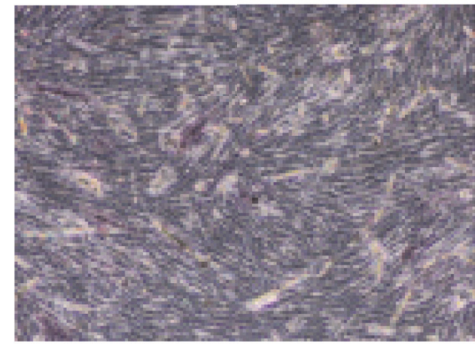

Overexpression

(d)

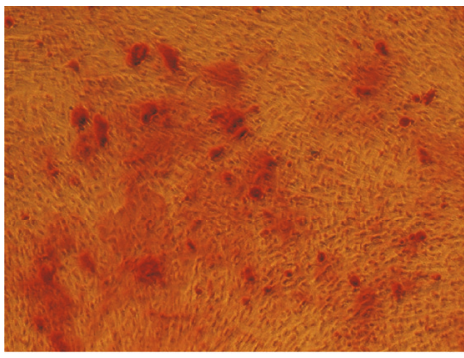

Control

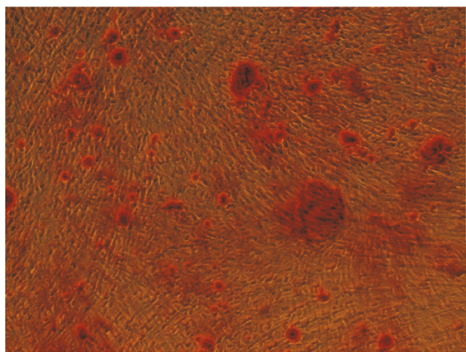

Control

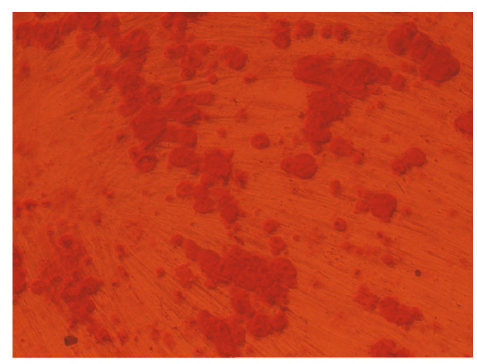

shRNA downregulation

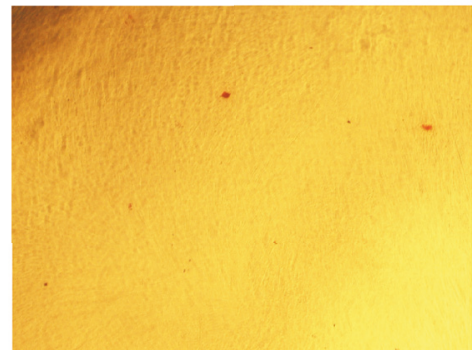

Overexpression

(e)

FIgURE 5: (a) The expression level of lncRNA ENST00000502125.2 was decreased during osteogenic differentiation of hBMmSCs. (b) ShRNA could significantly downregulate lncRNA ENST00000502125.2 expression. (c) Lentivirus vectors could significantly upregulate lncRNA ENST00000502125.2 expression. (d) Downregulation of lncRNA ENST00000502125.2 promoted the ALP staining intensity, and vice versa (200x). (e) Downregulation of lncRNA ENST00000502125.2 promoted Alizarin Red S staining intensity, and vice versa $(200 \mathrm{x}) .{ }^{* *}$ indicates $p<0.01$. 
ENST00000532315.1, and HIT000218960 for microarray validation was that these molecules were consistently upregulated or downregulated during osteogenic differentiation of hBMMSCs. Most of these lncRNAs and mRNAs were firsttime identified. GO analysis was performed to further annotate the biological function of differentially expressed mRNAs and a significant amount of GO terms were associated with developmental processes. Most enriched biological processes were correlated with osteogenic differentiation such as cartilage development, skeletal system development, and developmental process. For cellular component, ECM has been demonstrated to play an important role in regulating osteogenesis. For molecular function, integrin binding and growth factor activity are related with osteogenic differentiation. KEGG pathway analysis showed that many pathways such as cytokine-cytokine receptor interaction and ECMreceptor interaction were associated with osteogenic differentiation. To evaluate the genes regulated by lncRNAs, we selected two significantly deregulated lncRNAs (lncRNA ENST00000585537.1 and lncRNA eHIT000015952) for further analysis. In combination with the information of mRNAs, IncRNA ENST00000585537.1 was found to be located on chromosome 17 and three protein-coding genes (MAP2K6, TEKT1, and ABCA5) were near its $300 \mathrm{~kb}$ area. For lncRNA eHIT000015952, it was located on chromosome 3 and seven protein-coding genes (such as PAK2, MFI2, and DLG1) were near its $300 \mathrm{~kb}$ area (data not shown).

Through microarray analysis, we identified a number of lncRNAs potentially affecting osteogenic differentiation. LncRNA ENST00000502125.2 was chosen for further study as it was consistently downregulated during osteogenic differentiation of hBMMSCs, indicating that it might be a potent regulator of osteogenesis. LncRNA ENST00000502125.2 inhibition promoted the osteogenic differentiation of hBMMSCs and vice versa, suggesting it might play a critical role in osteogenesis. To the best of our knowledge, this was the first time to reveal this novel function of lncRNA ENST00000502125.2. Consistent with our findings, ectopic expression of lncRNA H19 promoted osteogenic differentiation of hMSCs both in vitro and in vivo, and opposite results were found when lncRNA H19 was inhibited. In addition, miR-675 was identified as a downstream target of lncRNA H19 [21]. Jin et al. reported that knockdown of lncRNA MIR31HG not only increased osteogenic differentiation of human adipose-derived stem cells both in vitro and in vivo, but also maintained this promotion effect even under the inflammatory environment, indicating targeting lncRNA MIR31HG might be an effective strategy to improve the osteogenic capacity of stem cells in bone engineering [13]. Similarly, lncRNA NONHSAT009968 suppression ameliorated staphylococcal protein A-inhibited osteogenic differentiation in hBMMSCs [22]. These studies demonstrate that lncRNAs are important regulators of osteogenic differentiation.

\section{Conclusions}

Collectively, our microarray data provides novel information regarding the potential role of mRNAs and lncRNAs in regulating the hBMMSC osteogenic differentiation. We also identify the top enriched GO and KEGG pathways. Moreover, lncRNA ENST00000502125.2 is demonstrated to be a negative regulator of osteogenic differentiation, indicating it might be a novel therapeutic target for promoting bone formation.

\section{Conflicts of Interest}

The authors declare no conflicts of interest.

\section{Authors' Contributions}

Xiaoling Qiu and Bo Jia contributed equally to this work.

\section{Acknowledgments}

This work was supported by the National Science Foundation (no. 81670950) and Guangdong Natural Science Foundation (no. 2015A030313787).

\section{References}

[1] M. E. Elsalanty and D. G. Genecov, "Bone grafts in craniofacial surgery," Craniomaxillofacial Trauma and Reconstruction, vol. 2, no. 3, pp. 125-134, 2009.

[2] M. Fröhlich, W. L. Grayson, L. Q. Wan, D. Marolt, M. Drobnic, and G. Vunjak-Novakovic, "Tissue engineered bone grafts: biological requirements, tissue culture and clinical relevance," Current Stem Cell Research \& Therapy, vol. 3, no. 4, pp. 254-264, 2008.

[3] M. Al-Nbaheen, R. vishnubalaji, D. Ali et al., "Human stromal (mesenchymal) stem cells from bone marrow, adipose tissue and skin exhibit differences in molecular phenotype and differentiation potential," Stem Cell Reviews and Reports, vol. 9, no. 1, pp. 32-43, 2013.

[4] L. Cui, S. M. Xu, D. D. Ma, and B. L. Wu, "The effect of TRPM7 suppression on the proliferation, migration and osteogenic differentiation of human dental pulp stem cells," International Endodontic Journal, vol. 47, no. 6, pp. 583-593, 2014.

[5] M. Mo, S. Wang, Y. Zhou, H. Li, and Y. Wu, "Mesenchymal stem cell subpopulations: phenotype, property and therapeutic potential," Cellular and Molecular Life Sciences, vol. 73, no. 17, pp. 3311-3321, 2016.

[6] L. Cui, S. Xu, D. Ma et al., "The role of integrin- $\alpha 5$ in the proliferation and odontogenic differentiation of human dental pulp stem cells," Journal of Endodontics, vol. 40, no. 2, pp. 235240, 2014.

[7] A. Gebler, O. Zabel, and B. Seliger, "The immunomodulatory capacity of mesenchymal stem cells," Trends in Molecular Medicine, vol. 18, no. 2, pp. 128-134, 2012.

[8] K. Kashi, L. Henderson, A. Bonetti, and P. Carninci, "Discovery and functional analysis of IncRNAs: methodologies to investigate an uncharacterized transcriptome," Biochimica et Biophysica Acta-Gene Regulatory Mechanisms, vol. 1859, pp. 315, 2016.

[9] J. Cao, "The functional role of long non-coding RNAs and epigenetics," Biological Procedures Online, vol. 16, article 11, 2014. 
[10] H. Lv, Y. Sun, and Y. Zhang, "MiR-133 is involved in estrogen deficiency-induced osteoporosis through modulating osteogenic differentiation of mesenchymal stem cells," Medical Science Monitor, vol. 21, pp. 1527-1534, 2015.

[11] O. Wapinski and H. Y. Chang, "Long noncoding RNAs and human disease," Trends in Cell Biology, vol. 21, no. 6, pp. 354361, 2011.

[12] W. Zhuang, X. Ge, S. Yang et al., "Upregulation of IncRNA MEG3 promotes osteogenic differentiation of mesenchymal stem cells from multiple myeloma patients by targeting BMP4 transcription," Stem Cells, vol. 33, no. 6, pp. 1985-1997, 2015.

[13] C. Jin, L. Jia, Y. Huang et al., "Inhibition of lncRNA MIR31HG promotes osteogenic differentiation of human adipose-derived stem cells," Stem Cells, In press.

[14] E. A. Gibb, E. A. Vucic, K. S. S. Enfield et al., "Human cancer long non-coding RNA transcriptomes," PLoS ONE, vol. 6, no. 10, Article ID e25915, 2011.

[15] Y. Huang, N. Liu, J. P. Wang et al., "Regulatory long non-coding RNA and its functions," Journal of Physiology and Biochemistry, vol. 68, no. 4, pp. 611-618, 2012.

[16] J. R. Evans, F. Y. Feng, and A. M. Chinnaiyan, "The bright side of dark matter: lncRNAs in cancer," Journal of Clinical Investigation, vol. 126, no. 8, pp. 2775-2782, 2016.

[17] S. Uchida and S. Dimmeler, "Long noncoding RNAs in cardiovascular diseases," Circulation Research, vol. 116, no. 4, pp. 737750, 2015.

[18] L. Wang, Y. Wang, Z. Li, Z. Li, and B. Yu, "Differential expression of long noncoding ribonucleic acids during osteogenic differentiation of human bone marrow mesenchymal stem cells," International Orthopaedics, vol. 39, no. 5, pp. 1013-1019, 2015.

[19] L. Malaval, A. K. Gupta, and J. E. Aubin, "Leukemia inhibitory factor inhibits osteogenic differentiation in rat calvaria cell cultures," Endocrinology, vol. 136, no. 4, pp. 1411-1418, 1995.

[20] S. Thysen, F. Cailotto, and R. Lories, "Osteogenesis induced by frizzled-related protein (FRZB) is linked to the netrin-like domain," Laboratory Investigation, vol. 96, no. 5, pp. 570-580, 2016.

[21] Y. Huang, Y. Zheng, L. Jia, and W. Li, "Long noncoding RNA H19 promotes osteoblast differentiation via TGF- $\beta 1 / \mathrm{Smad} 3 /$ HDAC signaling pathway by deriving miR-675," Stem Cells, vol. 33, no. 12, pp. 3481-3492, 2015.

[22] Y. Cui, S. Lu, H. Tan, J. Li, M. Zhu, and Y. Xu, "Silencing of long non-coding RNA NONHSAT009968 ameliorates the Staphylococcal protein A-Inhibited osteogenic differentiation in human bone mesenchymal stem cells," Cellular Physiology and Biochemistry, vol. 39, no. 4, pp. 1347-1359, 2016. 

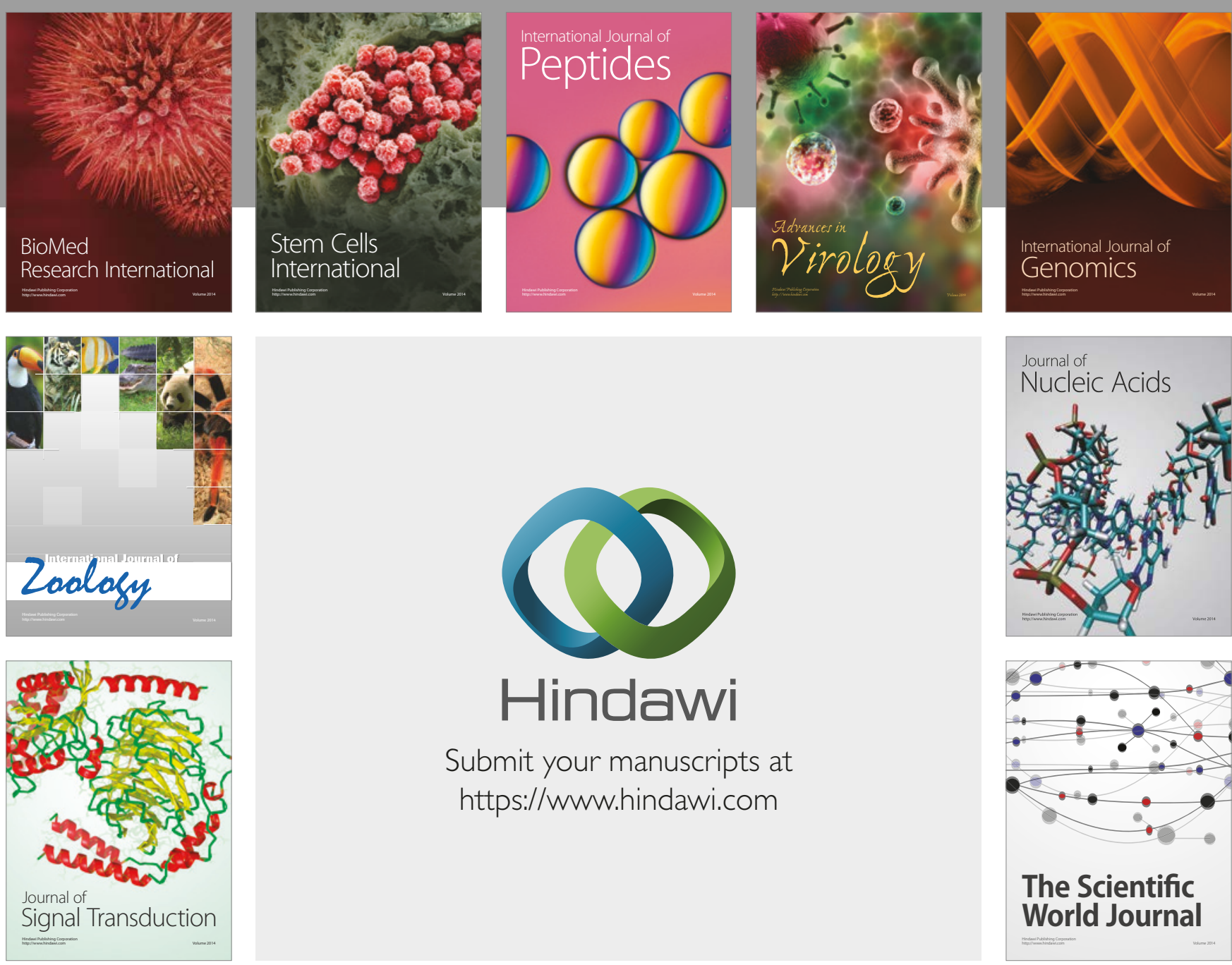

Submit your manuscripts at

https://www.hindawi.com
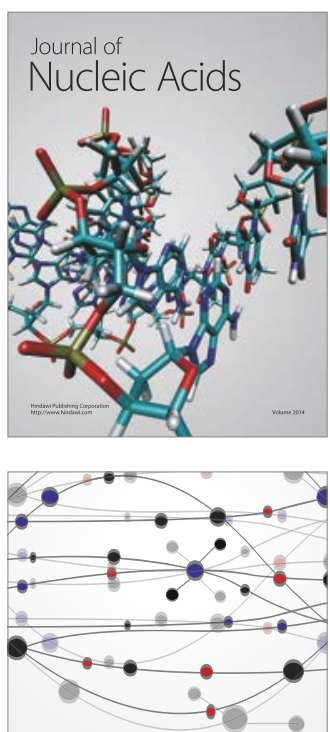

The Scientific World Journal

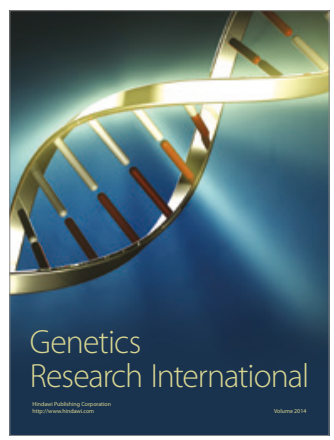

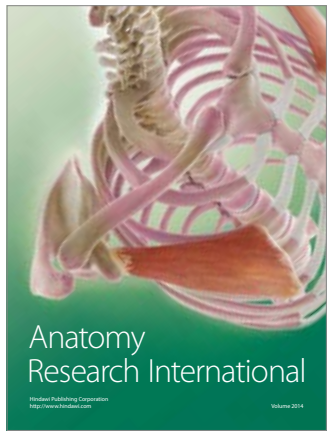

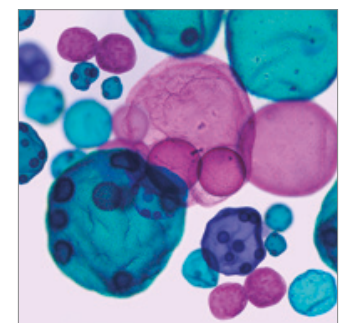

International Journal of Microbiology
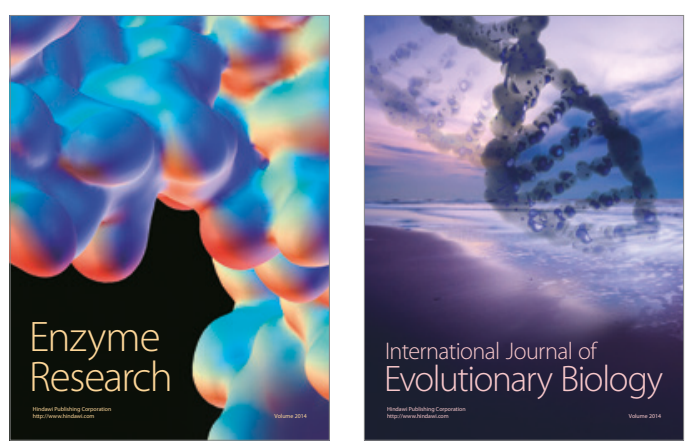
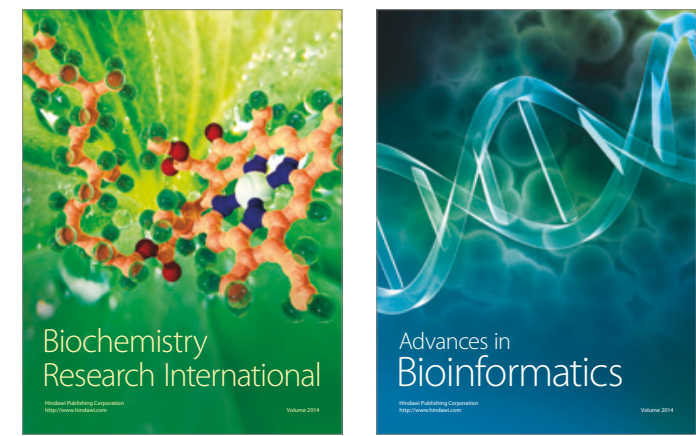

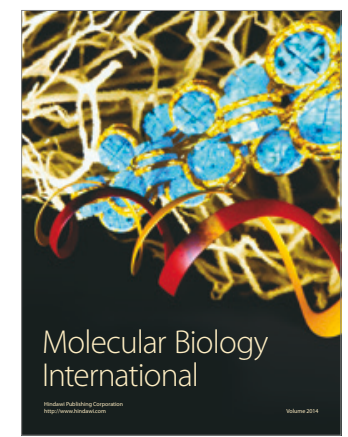

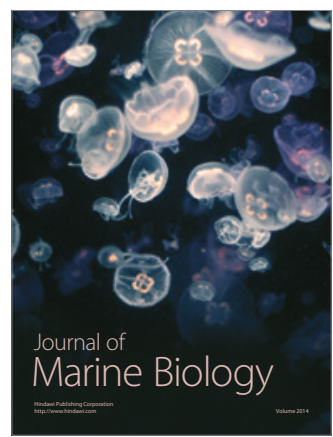

